DOI: $10.12731 / 2306-1561-2013-4-20$

\title{
ANALYSIS OF APPROACHES TO THE FORMATION OF FUNCTIONAL STRUCTURE OF AUTOMATED DATA PROCESSING SYSTEMS AND MANAGEMENT
}

\section{Chistyakova V.V., Morozova T.Yu.}

\section{Abstract}

The article examines the development of the functional structure of automated data processing systems and management (ACS). Given the concept of information system architecture and enterprise. Reviewed and analyzed by a variety of approaches to the design of automated data processing systems and management. Highlighted a number of shortcomings of traditional approaches, the main of which is the difficulty of their use in optimization problems of structure, the lack of the formation mechanism of the wood objectives automated system as a whole and its parts. It is proposed to solve the problem of formation of functional structure of automated data processing systems and management by analyzing a variety of purposes.

Keywords: information systems, control systems, optimization of the structure, a model of architecture, information architecture, software.

\section{УДК 004.9:681.3}

\section{АНАЛИЗ ПОДХОДОВ К ФОРМИРОВАНИЮ ФУНКЦИОНАЛЬНОЙ СТРУКТУРЫ АСОИУ}

\section{Чистякова В.В., Морозова Т.Ю.}

\section{Аннотация}

В статье рассматриваются вопросы разработки функциональной структуры автоматизированных систем обработки информации и управления (АСОИУ). Дается понятие архитектуры информаџионной системы и предприятия. Рассматриваются и анализируются различные подходы к проектированию АСОИУ. Вылелятся ряд недостатков традиционных подходов, основные из которых трудности их использования в задачах оптимизации структуры системы, отсутствие механизма формирования дерева целепологания автоматизированной системы в цүелом и ее элементов. Предлагается решать задачу формирования функииональной структуры АСОИУ на основе анализа множества иелей. 
МАТЕРИАЛЫ ІХ МЕЖДУНАРОДНОЙ ЗАОЧНОЙ НАУЧНО-ПРАКТИЧЕСКОЙ КОНФЕРЕНЦИИ МОЛОДЫХ УЧЕНЫХ

«ТЕОРИЯ И ПРАКТИКА ПРИМЕНЕНИЯ ИНФОРМАЦИОННЫХ ТЕХНОЛОГИЙ В ПРОМЫШЛЕННОСТИ И НА ТРАНСПОРТЕ», г. Москва, 12 ноября 2013 г.

Ключевые слова: проектирование информационных систем, системы управления, оптимизация структуры, модель архитектуры, информационная архитектура, программное обеспечение.

\section{Введение}

Для эффективного функционирования современным предприятиям требуется достоверная, оперативная информация, содержащая все необходимые сведения для принятия управленческих решений. Такая информация может быть предоставлена только в результате использования современных информационных технологий. Только системный подход к формированию стратегии развития архитектуры АСОИУ информационной, программной и технической, основанной на анализе потребностей предприятия в информационной поддержке процесса управления.

Существующие подходы к проектированию автоматизированных систем основываются на оценки существующих потребностей, не учитывая динамику развития бизнеса.

\section{Традиционная методика проектирования архитектуры АСОИУ}

Методика проектирования АСОИУ определяет процесс разработки информационной системы обеспечивающий создание системы, отвечающих целям и задачам организации, а также предъявляемым требованиям по автоматизации деловых процессов заказчика; гарантирующий создание системы с заданным качеством в заданные сроки и в рамках установленного бюджета проекта [1].

Требования к информационной системе со стороны заказчика - бизнес и пользовательские требования могут быть представлены в виде модели компонентов и объектов предметной области (рисунок 1).

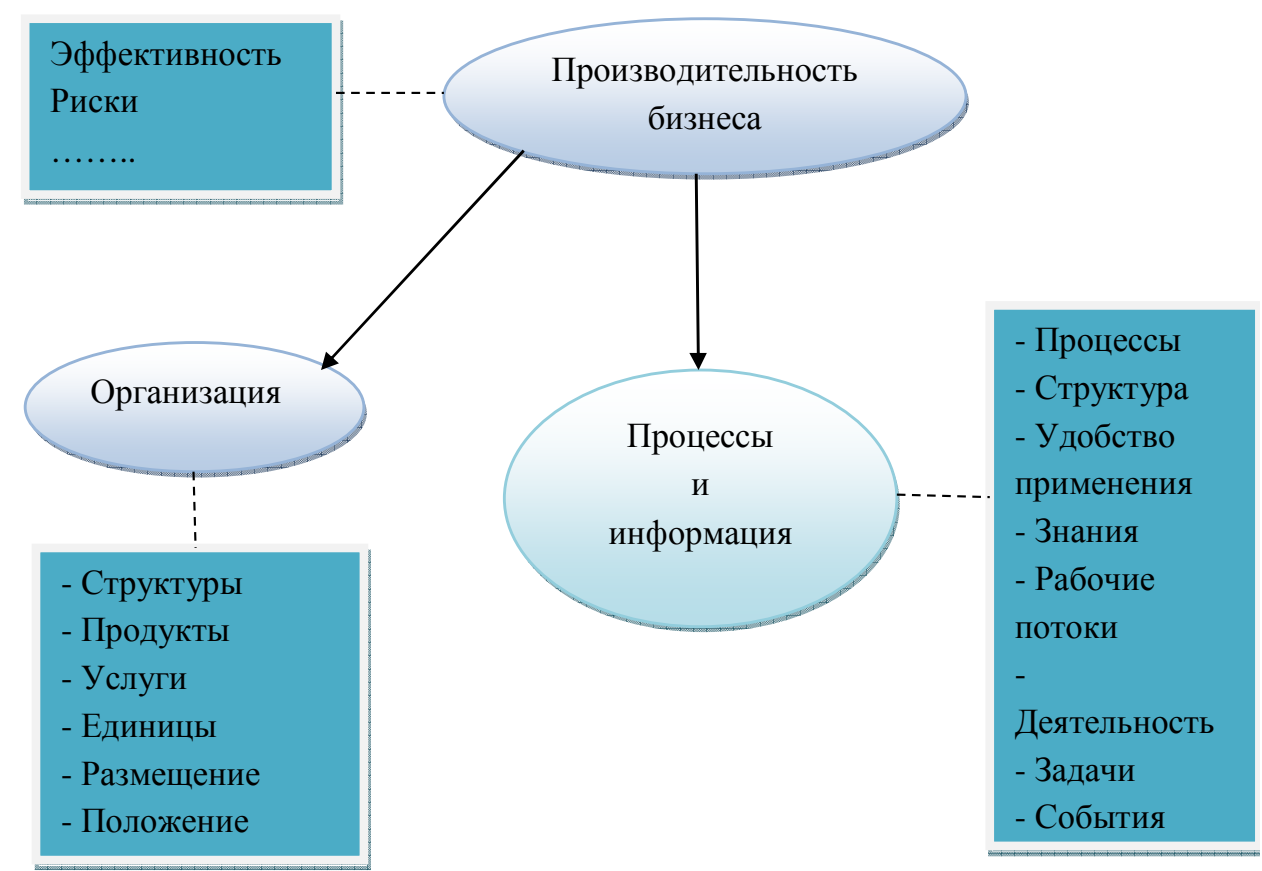

Рисунок 1 - Компоненты и объекты предметной области 
Наиболее значимыми элементами являются процессы и информация, организация, производительность. Элемент «процессы и информация» описывает и классифицирует бизнес-структуры, бизнес-процессы и потоки деятельности. Элемент «организация» описывает организационную структуру и методы работы, продукты и услуги, которые производятся на предприятии. Элемент «производительность» описывает экономические показатели эффективности работы предприятия.

Проектирование ИС охватывает три основные области:

- архитектура данных - проектирование объектов данных, которые будут реализованы в базе данных (рисунок 2);

- проектирование прикладных программ - архитектуры приложений (экранных форм, отчетов, которые будут обеспечивать выполнение запросов к данным и т.п.);

- технологическую архитектуру - учет конкретной среды или технологии, а именно: топологии сети, конфигурации аппаратных средств, используемой архитектуры (файл-сервер или клиент-сервер), параллельной обработки, распределенной обработки данных и т.п.

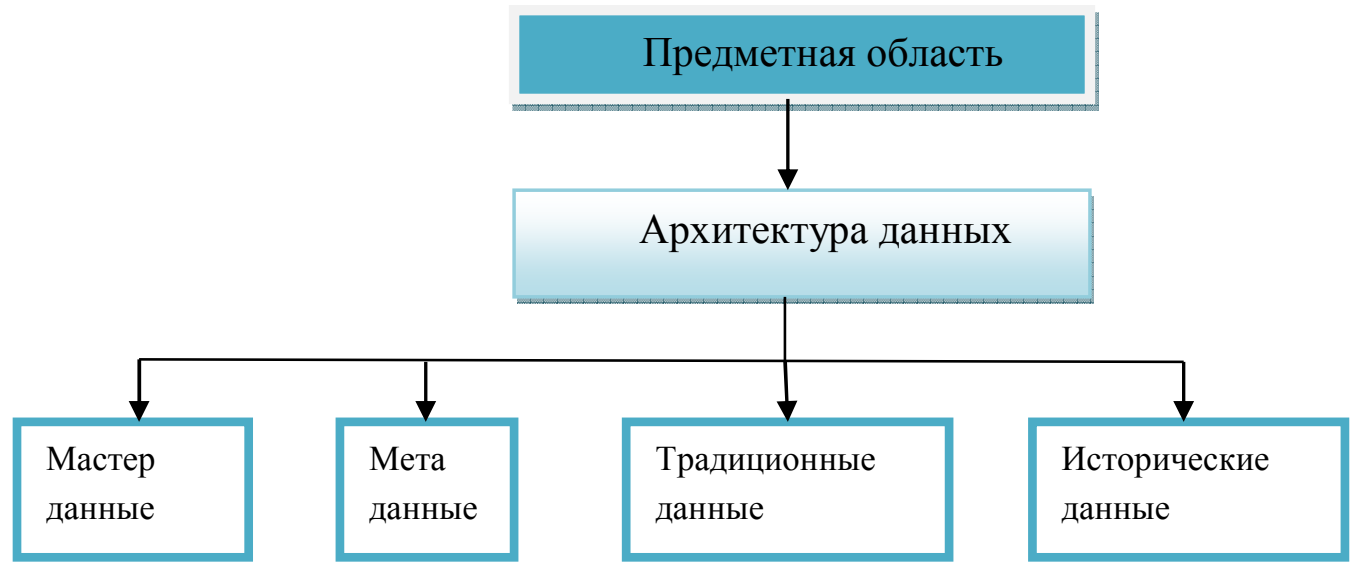

Рисунок 2 - Информационная архитектура

Архитектура приложений описывает классы приложений, с помощью которых осуществляется автоматизация предприятия и обработка информации. Приложения описываются без привязки к конкретным технологическим решениям. Классы приложений не столь динамично изменяются как технологии реализации. Архитектура приложений включает: классификацию приложений или предоставляемых сервисов и описание основных классов приложений используемых на предприятии; привязку прикладных программных продуктов к основным производственны процессам предприятия [2 - 5]; взаимодействие элементов программного обеспечения; функциональные требования к программному продукту.

\section{Анализ подходов к определению функциональной архитектуры АСОИУ}

Один из подходов к определению функциональной архитектуры основывается на использовании модели Захмана. Модель используется для отображения архитектуры 
предприятия и самой информационной системы. В данном случае модель отображает архитектуру в виде матрицы, строки которой представления о предприятии и выполняемых процессах сотрудников (руководитель, непосредственных исполнителей), участников проектного процесса (ИТ-руководителя, специалистов разрабатывающих обеспечивающие подсистемы). Столбцы матрицы отражают различные аспекты деятельности предприятия, сгруппированные на группы в виде ответа на вопросы: КТО? ЧТО? ГДЕ? КОГДА? КАК? ПОЧЕМУ? Каждая клетка матрицы содержит соответствующее описание конкретной стороны деятельности предприятия на конкретном уровне в виде определенной модели предметной области (возможно и текстового описания).

К недостаткам рассмотренной модели можно отнести трудности ее использования для решения задач оптимизации.

Другим средством формализации процесса проектирования АСОИУ является модель Спивака. Стивен Спивак соединил модель Захмана с методикой планирования и формирования архитектуры предприятия (Enterprise Architecture Planning, EAP). Модель архитектурного процесса приведена на рисунке 3.

Начало пла-

нирования
Уровень: 1

Начало процесса

\begin{tabular}{|c|l|}
\hline $\begin{array}{c}\text { Моделирование } \\
\text { бизнеса }\end{array}$ & $\begin{array}{l}\text { Используемые } \\
\text { системы и } \\
\text { технологии }\end{array}$ \\
\hline
\end{tabular}

Уровень 2:

Где мы сейчас находимся ?

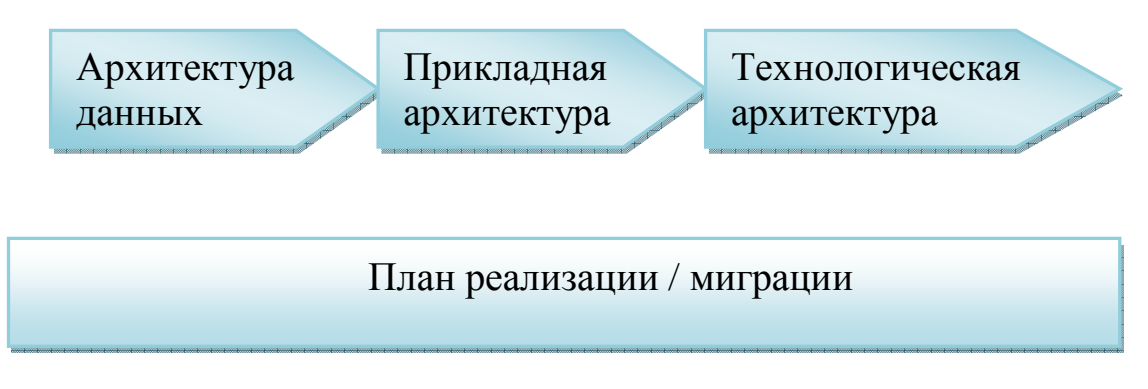

Уровень 3:

Чего мы хотим достичь

Уровень 4:

План как это осуществить

\section{Рисунок 3 - Модель Спивака}

Процесс работы с архитектурой ИС включает следующие шаги:

1. Определение общих контуров архитектуры.

2. Построение модели архитектуры «как есть».

3. Построение модели архитектуры «как должно быть». 
4. Определение шагов перехода от модели «как есть»к модели «как должно быть».

5. Формирование путей дальнейшего развития архитектуры.

При этом пятый шаг подразумевает формирование технологических проектных решений.

Одним из наиболее популярных методологий описания архитектуры является рамочная модель архитектуры, созданная The Open Group Architecture Framework (TOGAF). TOGAF является промышленным стандартом на описание архитектуры предприятия, который может быть бесплатно использован предприятием для разработки собственной архитектуры. Рамочная модель в качестве основных компонентов архитектуры рассматривает архитектуру бизнеса, информации, приложений и технологий. Однако делается акцент на процессы реализации проектных работ. Стандарт включает 9 фаз (рисунок 4).

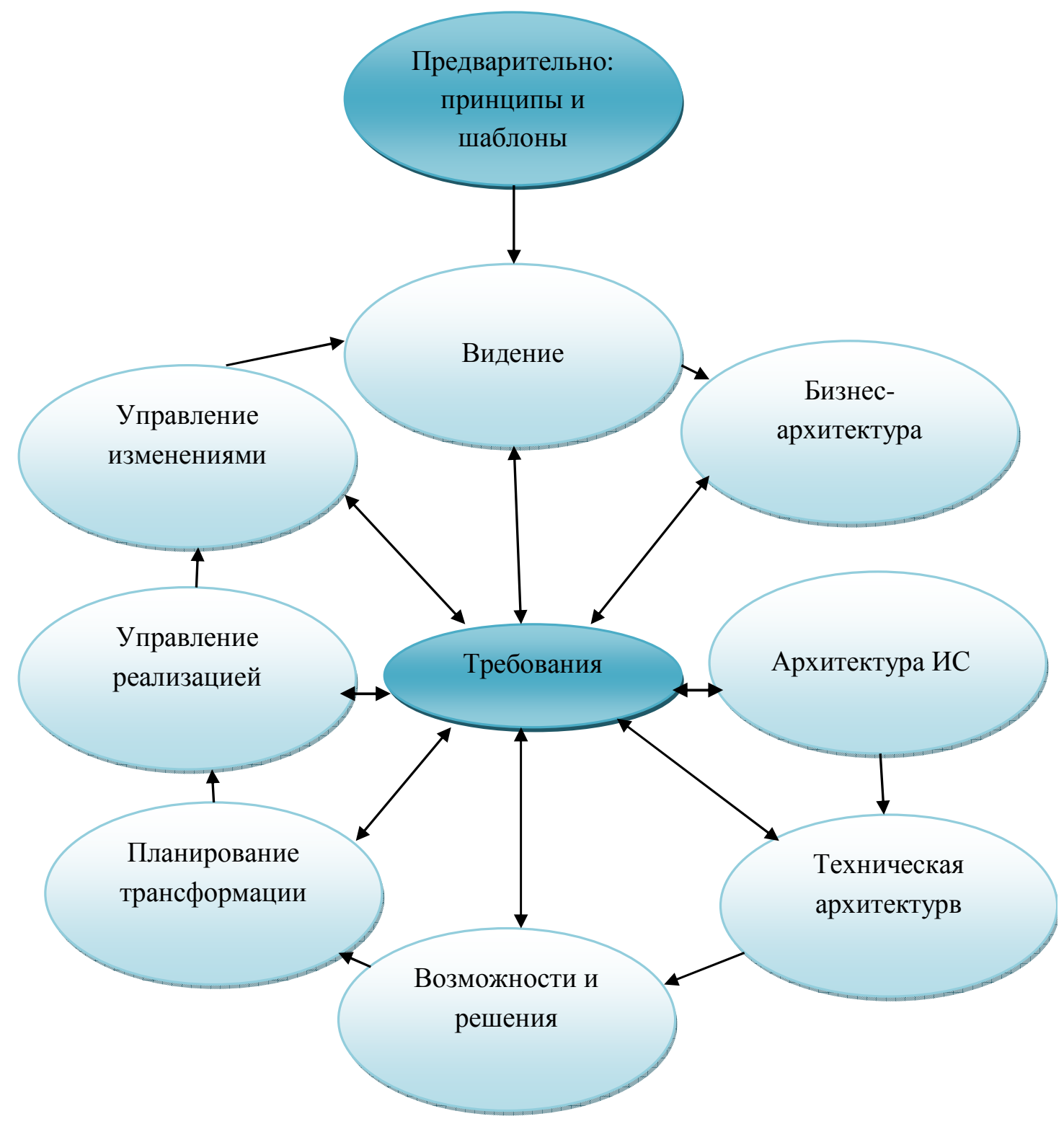

Рисунок 4 - Основные фазы TOGAF 
Каждая из фаз разбивается на этапы и работы и содержит перечень входных и выходных документов. Важной особенностью модели TOGAF является коллекция компоновочных блоков (шаблонов) и описание типичных выходов архитектурного процесса.

Анализируя рассмотренные подходы можно выделить невозможность использования рассмотренных моделей для решения задачи оптимизации функциональной структуры АСОИУ. Практически эти модели являются описательными и не предназначены для обоснованного принятия решения о структуре проектируемой информационной системы.

\section{Совершенствование процесса проектирования АСОИУ}

Автоматизированные информационные системы являются структурным элементов системы управления. Поэтому процесс их проектирования не может рассматривать вне связи с архитектурой-структурой системы управления и соответственно целью системы управления в целом, целью управляющей системы и ее элементов, целью объекта управления.

Таким образом, задачи определения функциональной структуры необходимо решать на множестве целей и множества структурных элементов системы управления. При этом эти множества являются упорядоченными и может быть представлено в виде дерева.

В рамках построения дерева целей и структуры объекта автоматизации и структуры информационной системы необходимо решить задачи нахождения содержательных характеристик и признаков структур ветвления дерева целей.

Круг исследуемых вопросов связан с исследованием следующих принципов:

- формирования законов, рекуррентного объяснения, минимаксного построения моделей;

- продуцирования;

- эквивалентности;

- структурной интеграции как обобщения структурно-функционального подхода для формирования дерева целей.

Адекватность построенных деревьев должна оцениваться по критериям:

- Морфологии дерева - адекватности системообразующей модели АСОИУ системе управления.

- Адекватности уровней дерева - характер рекурентности уровней дерева целей должно соответствовать числу и уровням системы управления.

- Минимальности ветвления - морфологические признаки ветвления дерева целей должны в минимальной степени отражать каждый из нарастающих уровней сложности процессов обработки информации.

Такой подход позволит всесторонне учесть потребительские требования к информационной системы со стороны основных бизнес-процессов. Поэтому при оптимизации, проводимой в процессе проектирования учитывается несколько 
параметров качества проектируемой системы (в частности, поддержка целей системы управления).

В процессе проектирования должны использоваться разнообразные методы формальная оптимизация, экспериментальные методы и моделирование различного вида, эвристика.

Методы формальной (математической) оптимизации наиболее пригодны именно на ранних стадиях проектирования (предпроектная стадия), когда приходится оперировать с достаточно общими (абстрактными) моделями. При этом желательно сформулировать предельные функциональные требования к проектируемой системе. В этом случае можно использовать критерии векторной оптимизации, критерии предпочтения - Паретто или Слейтера [6 - 10].

При проектировании АСОИУ приходиться сталкиваться с изменением объекта автоматизации. Учет произошедших изменений как фактической характеристики приводит к снижению качества информационной системы в период ее усовершенствования. Поэтому представляется целесообразным решать задачу прогноза [11 - 15] развития предприятия с целью корректировки дерева целей им дальнейшего опережающего структурного синтеза автоматизированной системы.

\section{Заключение}

Развитие предлагаемого подхода позволит разрабатывать АСОИУ соответствующие автоматизированной системе управления. А именно определять функциональную структуру автоматизированной системы в соответствии с целями системы управления и ее элементов. Кроме того, опережающее развитие информационной системы позволит сократить ожидание приложения структурами бизнеса.

\section{Список информационных источников}

[1] Морозова Т.Ю. Основные тенденции развития автоматизации передовых отраслей промышленности // Промышленные АСУ и контроллеры. 2010. № 12. С. 12-14.

[2] Чистякова М.А., Петриченко И.П. Вопросы разработки автоматизированного комплекса учета и контроля движения материальных объектов на промышленноторговых предприятиях с использованием RFID-технологии // Наукоемкие технологии. 2008. № 7. Т.9. С. 53-59.

[3] Чистякова М.А. Автоматизированный комплекс учета и контроля движения материальных объектов на промышленно-торговых предприятиях с использованием радиочастотной идентификации // Промышленные АCУ и контролеры. - 2009. №7. С. 23-28.

[4] Скворцова Т.И. Автоматизированное рабочее место материально-ответственного лица // Промышленные АСУ и контроллеры. - 2009. № 8. С. 8-11.

[5] Рязанов Д.А., Скворцова Т.И. Автоматизация одной из сфер деятельности предприятий общественного питания // Сборник научных трудов Sworld. 2007. T. 2. № 1. C. $80-83$. 
[6] Остроух А.В. Основы построения систем искусственного интеллекта для промышленных и строительных предприятий: монография / А.В. Остроух. - М.: ООО «Техполиграфцентр». - 2008. - 280 с. - ISBN 978-5-94385-033-2.

[7] Остроух, А.В. Интеллектуальные системы в науке и производстве: учеб. пособие / А.В. Остроух, А.Б. Николаев. - Palmarium Academic Publishing. Saarbrucken, Germany. - 2012. - 312 p. - ISBN 978-3-659-98006-0.

[8] Остроух, А.В. Информационные технологии в научной и производственной деятельности / [ред. А.В. Остроух] - М: ООО "Техполиграфцентр", 2011. - 240 с. ISBN 978-5-94385-056-1.

[9] Остроух, А.В. Ввод и обработка цифровой информации: учебник для нач. проф. образования / А.В. Остроух. - М.: Издательский центр «Академия», 2012. - 288 с. - ISBN 978-5-7695-9457-1.

[10] Васюгова С.А. Исследование перспектив и проблем интеграции человека с компьютером: искусственный интеллект, робототехника, технологическая сингулярность и виртуальная реальность / А.В. Остроух, С.А. Васюгова, М.Н. Краснянский, А. Самаратунга // Перспективы науки. - 2011. - № 4(19). - С. 109 112.

[11] Маценко К.В., Никонов В.В. Подготовка данных для автоматизированного анализа // Сборник научных трудов SWORLD. 2007. № 1. T.2. C 19-22.

[12] Барский А.Б. Нейронные сети: распознавание, управление, принятие решений. М.: Финансы и статистика, 2004.

[13] Никонов В. В Решение задачи прогнозирования на основе аппарата искусственных нейронных сетей // Сборник научных трудов SWORLD. 2007. №1. T.2. C 7-10.

[14] Морозова Т.Ю., Сумкин К.С. Модели контроля доступа пользователя к ресурсам системы //Сборник научных трудов Sworld. 2007. №1. Т.2. С. 11-14.

[15] Морозова Т.Ю., Петров О.М. Вероятностно-статистические методы и средства повышения эффективности защиты и обработки информации в беспроводных сетях. М., 2008. 